\title{
An Overview of Plant Immunity
}

\section{Doughari $\mathrm{JH}^{*}$}

Department of Microbiology, School of Pure and Applied Sciences, Modibbo Adama University of Technology, P.M.B 2076, Yola, Nigeria

\begin{abstract}
The exposure of plants in open land and to various harsh environmental conditions means that they are at risk of being attacked by different types of microbial pathogens. For survival strategies, the plants interact with microbes in different ways, many of whom result in disease conditions. Because plants are sessile, it means that they must continuously integrate both biotic and abiotic signals from the environment which also means they must be able to distinguish those signals that are harmless from those that are potentially harmful. Consequently, plants have evolved a variety of adaptive mechanisms that provides them with basal immune natural protective measures to fight against these microbial pathogens, pests and other vertebrate diseases. Recognition of these pathogens is made possible by certain group of physiological elicitors that activates microbial or pathogen recognition proteins (MAMPs or PAMPs) of the basal immunity that enables the plant to recognize the invading pathogens. However, because the plant immune system lack circulating cells such as antibodies and macrophages of animals, the signal responses are partitioned both locally over several cell diameters and systemically in a limited sphere. Adequate understanding of the cell signaling mechanisms and the role of hormones in disease resistance will assist in developing a very effective control measure against plant diseases for more productive agricultural turn over.
\end{abstract}

Keywords: Basal immunity; Hypersensitive response; Microbial associated molecular proteins; Pathogen recognition; Hemibiotrophs

\section{Introduction}

One of the major challenges for a very rapidly growing world population is the need to meet up the demand for adequate food supply. This requires ecologically sound, compatible strategies in agriculture for sustainable crop production. However emerging and re-emerging plant diseases continuously challenge this ability; consequently [1], the control of plant diseases continues to be an uphill task.

Plant diseases or phyto-pathogens (bacteria, fungi, viruses, and pests) affect plants right from the planting stage up to harvesting and storage of the produce. For plants to survive the harmful effects of those antagonistic interactions, they must overcome the pathogenic mechanisms that results in the disease process. Consequently, they have evolved several different defence mechanisms by which they defend themselves against these microbial infections $[2,3]$. These defence mechanisms or immunity is termed basal resistance, a term referring to two distinct aspects of plant-pathogen interaction: constitutive (physical barriers to pathogens) and induced (non-specific pathogen elicited) defences [4,5].

\section{Mechanisms of Plant-microbe Interactions and Plant Disease Formation}

Plants constitute an excellent ecosystem for microorganisms. This means that there are both mutualistic and pathogenic plantmicrobe interactions [1]. Plant-microbe interactions occur externally through the establishment of rhizosphere, root colonization, and nutrient acquisition. The rhizosphere, the particulate matter and microorganisms that cling to roots, is formed via mechanisms including motility, attachment by microbes and efficient nutrient supply by the plant root. Roots exude high molecular weight compounds like sugars, amino acids, organic acids, phenolics, fatty acids, and enzymes into the rhizosphere. Organic acids and sugars act as carbon source while as amino acids act as a source of nitrogen. Root exudes alter both physical as well as chemical characteristics of soil that in turn contribute in making soil a suitable habitat for microbial community to flourish $[1,4]$.
Phytopathogens are mainly grouped into three based on their modes of plant infection: necrotrophs, biotrophs and, hemibiotrophs. All microbial pathogens that attack and kill their host feed on the hosts' nutritive materials are described as necrotrophs (e.g. the gray mold fungus Botrytis cinerea and the bacterial soft-rot pathogen Erwinia carotovora that). For those pathogens that live their host to continue their life cycle as they continue to feed on its nutritive material, they are referred to as biotrophs (e.g. powdery mildew fungus Blumeria graminis and Cladosporium fulvum and the bacterial rice pathogen Xanthomonas oryzae). And for those pathogens that require a living host initially, but kill it at later stage of infection, such pathogens are described as hemibiotrophs (e.g. Magnaporthe grisea, the causative agent of rice blast disease). In general, bacteria and fungi adopt either a biotrophic or necrotrophic mode of infection while viruses are ideal biotrophs although viral infection can consequently result in host cell death [6-9].

\section{Conditions Under Which a Pathogen Causes Disease in Plants}

\section{Host, pathogen and environment}

A plant disease is said to occur a susceptible plant described as compatible host is infected by a pathogen under environmental conditions that favor disease, in other words there must be a successful interaction between the three components; pathogen, host and environment, the three factors are interconnected in a disease process in a pyramid [10]. In the case of the host, disease occurs when host

*Corresponding author: Doughari $\mathrm{JH}$, Department of Microbiology, School of Pure and Applied Sciences, Modibbo Adama University of Technology, P.M.B 2076, Yola, Nigeria, Tel: +2347035599712; E-mail: jameshamuel@yahoo.com

Received December 02, 2015; Accepted December 10, 2015; Published December 15, 2015

Citation: Doughari JH (2015) An Overview of Plant Immunity. J Plant Pathol Microbiol 6: 322. doi:10.4172/2157-7471.1000322

Copyright: (c) 2015 Doughari $\mathrm{JH}$. This is an open-access article distributed under the terms of the Creative Commons Attribution License, which permits unrestricted use, distribution, and reproduction in any medium, provided the original author and source are credited. 
pre-exisiting defence mechanism are not enough to check the entry of pathogen and the range of plant species on which a pathogen is capable of causing disease is described as host range, and for the pathogen, it elicits disease when it avoids timely eliciting active defence system in plant tissue or habits active defence response by secreting metabolic toxins and for the environment, favourable environmental factor is required for the pathogen and unfavourable environment for the host, otherwise even the most susceptible plant exposed to huge amounts of pathogen will not develop disease $[10,11]$.

The potential for any phytopathogen to cause disease also depends on presence or absence of; recognition factors, host receptors and sites for toxins as well as, availability of essential nutrients and growth factors $[5,12]$.

\section{Microbe-host compatibility and incompatibility}

For any pathogen to cause disease in a plant host, it is said that the interaction between the two must be compatible. Compatible interactions only occur when the bacterium infects susceptible host plants causing disease symptoms Incompatible interactions occur when the bacterium encounters a non-host or a resistant host plant (cultivar-specific resistance, or non-host resistance) $[3,12,13]$.

\section{Recognition factors}

The first step in infection process is the cell-to-cell communication between host and pathogens [14]. Plants of species or varieties may not be infected by pathogen if their surface cells lack specific recognition factors. If the pathogen does not recognize the plant as one of its hosts it may not adhere to the host surface or it may not produce infection substances such as enzymes, or structures (appresoria, haustoria) [4]. These recognition molecules are of various types of oligosaccharides and polysaccharides and glycoproteins.

\section{Host receptors and sites for toxins}

Host specific toxins produced by the parasite accounts for the pathologic manifestations in majority of host parasite interactions. The toxin molecules attach to specific sensitive sites or receptors in the cell. Only the plants that have such sensitive sites are susceptible and become diseased [15].

\section{Essential nutrients and growth factors}

Many facultative saprophytes and most of the obligate parasites are host specific. Sometimes they are so specialized that they can grow and reproduce only on certain species. This suggests that for these pathogens the essential nutrients and growth factors are available only in these hosts. Absence of these nutrients and stimulus make the other varieties and species unsuitable hosts $[15,16]$.

\section{Phtytopathogen Infection Processes}

For a pathogen to successfully infect a plant, four processes must be undergone; i) it must find the host and the appropriate entry site, ii) it must develop specialized infection structures, ii) it must overcome preformed and induced host defenses and iv) it must take up nutrients, grow, and colonize host tissue. In addition, morphological changes by the pathogen are essential to penetration and adaptation to plant tissue. Access into plant tissues can either be by direct penetration of plant surfaces or by entrance through physical injuries or natural openings such as the stomata $[17,18]$. Penetration of entry into plant tissues follows successful colonization of plant surfaces. Different microbial groups; bacteria, fungi and viruses, have varying mechanisms by which they gain access to plant tissues [19]. Once inside the plant host, pathogens proliferate in the plant apoplast (the intracellular spaces of plants) from where they absorb the host plant nutrients. In the apoplast, pathogens usually inject so-called Type III secreted effectors (T3SEs) into plant cells by using a Type III secretion system (T3SS). The secreted T3SEs attribute to the pathogenesis of plant pathogens through disturbing normal physiological and biochemical processes of plants $[20,21]$.

\section{Bacteria}

Bacteria as plant pathogens can cause severe economically damaging diseases, ranging from spots, mosaic patterns or pustules on leaves and fruits, or smelly tuber rots to plant death. Some cause hormonebased distortion of leaves and shoots called fasciation or crown gall, a proliferation of plant cells producing a swelling at the intersection of stem and soil and on roots. Infection of plants by bacteria can occur in multiple ways. Infection is generally considered to be passive, i.e., accidental, although a few cases of plant chemoattractants have been reported. Bacteria can be sucked into a plant through natural plant openings such as stomata, hydathodes or lenticels. They can enter through abrasions or wounds on leaves, stems or roots or through placement by specific feeding insects. Artificially, bacteria are most commonly introduced into plants by wounding, by pressure-driven aerosols mimicking wind-driven rains, vacuum infiltration, or by seed immersion into inoculum [22].

Bacterial endophytic entry into plant tissues begins with colonization of the plant body. For colonization to occur, the bacterial cell must be brought into close proximity with the cell, and that is made possible by various tactic responses such as aerotaxis (towards plant atmosphere), chemotaxis (towards plant chemicals such as isoflavones, amino acids, sugars and other exudates) and even electrotaxis towards weak electric fields generated by roots, moving the bacterial cells towards the host plant tissue [14]. For successful colonization to occur, bacteria must successfully attach to the plant surface. Attachment to plant parts achieved with the help of pilli, cell surface proteins, and polysaccharides. $\mathrm{O}$-antigen side chain of outer membrane lipopolysaccharides have been found to be associated with adhesion of cells to surfaces including plants $[17,23]$.

Once colonized, bacteria enter into plant tissues through such natural openings as trichomes, lenticels, stomata, nectarthodes, stigma, hydathodes, lateral root or through wounds. Once inside the plant system, bacteria proliferate the apoplast, that is, the intercellular spaces or xylem vessels. Invasion of the apoplast results in parenchymatous and vascular or parenchymatous-vascular diseases [14]. Some P syringae strains make coronatine, a jasmonic acid mimic that suppresses salicylicacid-mediated defence to biotrophic pathogens and induces stomatal opening, helping pathogenic bacteria gain access to the apoplast $[2,22-$ 24].

Besides the endophytic habitat, some bacterial species also have the capacity to survive as epiphytes on plant surfaces (phylloplane, rhizoplane, carpoplane, etc.), predisposing the plant to rapid infection under favourable conditions [13]. Many bacteria produce siderophores which are iron-binding ligands that enable them grow and multiply on plant surfaces. Ferric $\left(\mathrm{Fe}_{3}\right)$ form of iron required by microbes, is present in soil in a very small amount (1018 M at $\mathrm{pH} 7.4)$, too low to support growth of microorganisms having need of iron in the concentration of $106 \mathrm{M}$. The siderophores are extracellular, low molecular weight microbial compounds that have the ability to make ferric form of iron available for the micro-organism. They actively sequester iron with high affinity and transfer it to the interior environment of cell. Siderophores acquire the iron from insoluble hydroxides or from iron adsorbed to solid 
surfaces, they can also extract iron from iron complexes, such as ferric citrate, ferric phosphate, Fe-transferin, ferritin, or from iron bound to sugars, glycosides, or even from iron chelators like EDTA by ferric/ ligand exchange reactions. Fungal siderophores, have lower affinity for iron and therefore plays an insignificant role in fungal binding to surfaces. Environmental factors that modulate siderophore production include, concentration and the form of iron, degree of aeration, adequate supply of carbon, nitrogen, and phosphorus, $\mathrm{pH}$, and light in addition to presence of trace elements such as magnesium, zinc, and molybdenum [1]. Factors predisposing plants to bacterial infections include favourable nutrient conditions, favourable susceptible parts e.g. flowers or roots as well as wind-driven rain carriage of bacterial inoculum [22].

\section{Fungi}

Fungi can directly enter plant epidermal cells, or extend hyphae on top of, between, or through plant cells. Many fungal or oomycete pathogens in contact with plant surface and plant tissue environment develop highly specialized infection structures. Two important feeding structures produced by the fungi to invade the host cell plasma membrane are appressoria and haustoria [18].

Appressoria has tough melanin-pigmented cell wall which plays an important role in the infection process as non-melanin mutants are found to be non-pathogenic The appressoria accumulates molar concentrations of glycerol in the melanin layer that produces hydrostatic turgor which can puncture plant cell membranes thereby facilitating the invasion process. Infection process by appressoria can involve enzymatic action. External matrix around appressoria contains cutinase, cellulases, and other enzymes to help soften the cuticle, thereby aiding adhesion and penetration of the host cell wall by fungal hypha. The hypha then enters the leaf epidermal cell and gets differentiated into bulbous and lobed infectious hypae, which grows intra - and intercellularly. Example of appressoria producing fungi is the rice blast fungus Magnaporthe grisea which produces a domeshaped-appressorium that perforates the surface of a rice leaf thereby breaching the thin but tough rice leaf cuticle to invade the leaf and cause disease $[18,26]$.

Haustoria formed within living cells of host organism by other fungi, oomycetes and parasitic plants are assumed to play a major role in the absorption of plant metabolites [27]. They serve as transporters of important nutrients such as amino acids thereby facilitating the dissemination of infections, but because they are intimately associated with plant cells, they are difficult to study. A few biotrophic fungi such as Cladosporium fulvum do not form haustoria but instead grow exclusively in apoplast, subsisting on leaked nutrients [2].

\section{Viruses}

Viral entry is only possible through physical injuries induced either by environmental factors or by vectors [28]. Once inside the cell, the virus mobilizes locally and systematically through intracellular (symplastic) movement through the plasmodesmata, which may occur in either virion or non-virion form. Movement proteins (MPs) and other virus-encoded factors assist virus movement inside the plant system in a coordinated and regulated manner, by acting together with the host components. Vector mediated viral infection is facilitated by sap-sucking species of arthropods, which can deliver virus particles directly into the vasculature and thus rapidly disseminating the virus throughout the plant $[29,30]$.

\section{Nematodes and aphids}

These feed by inserting a stylet directly into a plant cell. Pathogens produce small molecule effectors that mimic plant hormones [2,24,25].

\section{Plant Defence Mechanisms against Pathogens}

Although lacking an immune system comparable to animals, plants have developed an innate immunity comprising several structural, chemical, and protein-based defenses designed to detect and stop invading organisms (microbes, pests and herbivores). These defence mechanisms can be grouped into two: pre-existing and post-existing [19].

\section{Pre-existing-structural defence mechanisms}

The pre-existing, preformed, passive or pre-invasive plant defence mechanisms are the innate basal first line immune defence gadgets indigenously constitutive in the plant even before colonization by the pathogen. The basal structural defense mechanisms often found on the plant surface, are generally of the categories that present physical barriers to pathogen entry [27]. The physical barriers include wax layers, rigid cell walls or, cuticular lipids, cutin, bark etc. These structures not only protect the plant from invasion, they also give the plant strength and rigidity and exist as integral component physiological structures throughout the lifespan of the plant [4].

The wax layer and cuticle: Waxes are mixture of long chain aliphatic compounds which prevent the retention of water on plant surface essential for spore germination. Because the epidermal cells of aerial plant parts are often covered in a waxy cuticle that is made up of fatty acids, leaf surfaces are always negatively charged. This charge often repels airbone spores or propogules of several microbes from settling on them. Thus in addition to prevention of water loss from the plant, the fatty acid layer also prevents microbial pathogens from coming into direct contact with epidermal cells thereby limiting infection [31]. In aquatic plants, the cuticle is relatively thin, while in xerphytes such as the cactus, it is extremely thick. The hydrophobic nature of the cuticle also prevents water from collecting on the leaf surface, an important defense against many fungal pathogens that require standing water on the leaf surface for spore germination. The cuticle covers consists of pectin layer, a cutinized layer and a wax layer. Cutin is composed of fatty acids. Only few pathogens are known to dissolve cutin enzymatically. For instance Fusarium solani produce cutinases that degrade the cuticle and allow the fungi to penetrate the epidermis [3].

Epidermal layer: Epidermis is the first layer of living host cells that comes in contact with attacking microbes. It is the first line of defense against invading pathogens and consists of both specialized and unspecialized cells. The toughness of epidermis is due to the polymers of cellulose, hemicelluloses, lignin mineral substances, polymerized organic compounds, suberin etc., [32]. Potato tubers resistant to Pythium debaryanum contain higher fibre. Silicon accumulation in epidemal walls provides resistance against fungal attack. Suberization of epidermis confers protection against plant Xanthomonas axonopodis pv. Citri because of broad cuticulat lips covering the stomata. A functional defence mechanism has been observed in some varities (cvHope) in which tomato open late in the day when moisture on leaf surface has dried and the infection tunes have become nonfunctional $[33,34]$.

The epidermis 'constitutes the outermost protective tissue system of leaves, floral parts, fruits, seeds, stems, and roots of plants until 
they undergo considerable secondary growth. In woody plants, the periderm or outer bark replaces the epidermis on stems and roots and is often more thicker due the presence of high amounts of waterresistant suberin. It is an excellent example of a preformed structural barrier which prevents many pathogens and insects from reaching the living cells underneath [35].

Cytoskeleton: Actin cytoskeleton is the fundamental structural component of eukaryotic cells. The actin cytoskeleton plays an important role in formation of physiological barrier in the site of infection. It is involved in the vesicular trafficking responsible for transport of fortifying cell wall components, callose and, antimicrobial compounds to the site of infection. The plant cytoskeleton is an important obstacle encountered by pathogens. Disruption of the actin cytoskeleton of barley Hordeum vulgare L.), wheat, cucumber (Cucumis sativus L.) and tobacco (Nicotiana tabacum L.) resulted in cellular penetration of the crops by several non-host fungi and in the defense against pathogenic bacteria $[35,36]$.

Hydathodes: Hydathodes or water-exuding pores are natural openings on the edges of leaves that serve to excrete excess water from the interior [23]. They are easy entry pints of bacterial pathogens such as Xanthomonas campestris pv. campestris (black rot of cabbage), Plants also possess nectarthodes similar to hydathodes, except that they secrete sugary nectar which serves as barrier to sugar intolerant microbes. In addition to these openings, leaves are lined with leaf hairs which also serve as obstacles pathogen entry. High hairlines of leaves and pods in chickpea confers resistance to Ascachyta rabei invasion. Groundnut varieties showing resistance to Cercospora leaf spots have thick epidemis-cum cuticle and compact paslisade layer, few and smaller stomata and high frequency or trochomes on the abaxial surface of leaf $[23,37]$.

Lenticles: Lenticles are opening in outer walls involved in gaseous exchange. They are weak points in defence unless the cork cells within them are suberized. After suberizatoin and periderm formation, lenticels are more resistant to invasion by pathogens [23].

Cell wall: The cell wall is a major line of defense against fungal and bacterial pathogens providing an excellent structural barrier. Structurally, it incorporates a wide variety of chemical defenses that can be rapidly activated when the cell detects the presence of potential pathogens. All plant cells have a primary and secondary cell wall, with the former giving structural support and is essential for turgor pressure, and the later developing inside of the primary cell wall after the cell's active growth has stopped [12]. Structurally, the primary cell wall is made of mainly cellulose, a complex polysaccharide consisting of thousands of glucose monomers linked together to form long polymer chains. These chains are bundled into fibers called microfibrils, which give strength and flexibility to the wall.

The cell wall may also contain two groups of branched polysaccharides: cross-linking glycans and pectins. Cross-linking glycans include hemicellulose fibers that give the wall strength via cross-linkages with cellulose. Pectins form hydrated gels that help "cement" neighboring cells together and regulate the water content of the wall. Soft-rot pathogens often target pectins for digestion using specialized enzymes that cause cells to break apart causing brown and "mushy" appearance in fruits or vegetables [4].

Many cell walls also contain lignin, a heterogeneous polymer composed of phenolic compounds that gives the cell rigidity. Lignin is the primary component of wood, and cell walls that become "lignified" are highly impermeable to pathogens and difficult for small insects to chew. Cutin, suberin, and waxes are fatty substances that may be deposited in either primary or secondary cell walls (or both) and outer protective tissues of the plant body, including bark [12].

Guard cells: Guard cells regulate gas exchange through small openings called stomata. Gaseous carbon dioxide gains access into the leaf through the stomata for use in photosynthesis while restricting excessive water loss from the plant. Stomatal pore size is highly regulated by plants, and guard cells can participate in defense by closing in response to the presence of MAMPs [2].

Trichomes: Trichomes also called "leaf hairs" are specialized epidermal cells mainly found on aerial plant parts. They give both physical and chemical protection against insect pests. The velvety appearance of dusty miller (Senecio cineraria) is caused by thousands of tiny trichomes covering the plant's surface [38]. Trichomes on the surface of soybeans (Glycine max) prevent insect eggs from reaching the epidermis. They also starve the insect larvae after hatching. The hookshape of snap bean (Phaseolis vulgaris) trichomes impale caterpillars as they move across the leaf surface, and glandular trichomes in potato and tomato secrete oils that repel aphids [2].

Thorns: Thorns are modified branches that protect plants from grazing vertebrates, and include the honey locust tree (Gleditsia triacanthos). Many cacti produce thorn-like structures that are actually modified leaves or parts of leaves (e.g. stipules) called spines which serve similar purposes, such as in the barrel cactus (Ferocactus spp.). In some plants, such as the rose plant (Rosa spp.) botanically speaking, the "thorns" on the stem are neither true thorns nor spines, but are actually outgrowths of the epidermis called prickles [3]. They however serve essentially the same role as thorns.

Idioblasts: Also referred to as "crazy cells", idioblasts are highly specialized immune plant cells. They help protect plants against herbivory because they contain toxic chemicals or sharp crystals mainly calcium oxalate which tear the mouthparts of insects and mammals as they feed [39].

There are many classes of idioblasts including pigmented cells, sclereids, crystalliferous cells, and silica cells. Pigmented cells often contain bitter-tasting tannins that make plant parts undesirable as a food source [39]. Young red wines often contain high levels of tannins that give wine a sharp, biting taste. Sclereids are irregularly-shaped cells with thick secondary walls that are difficult to chew: the rough texture of pear fruit (Pyrus spp.) is caused by thousands of sclereid stone cells that can abrasively wear down the teeth of feeding animals. Stinging nettles (Urtica dioica) produce stinging cells shaped like hypodermic needles that break off when disturbed and inject highly irritating toxins into herbivore tissues. Some stinging cells contain prostaglandins, hormones that amplify pain receptors in vertebrate animals and increase the sensation of pain [2].

Crystalliferous cells contain crystals of calcium oxalate that may tear herbivore mouthparts when chewed and can be toxic if ingested. Members of the genera Philodendron and Dieffenbachia are very common tropical house plants that contain large amounts of these cells. Humans and pets who chew the leaves of these plants may experience a burning sensation in the mouth and throat that is often accompanied by swelling, choking, and an inability to speak. For these reasons, species of Dieffenbachia are commonly called dumb cane. Grasses and sedges contain rows of silica cells in their epidermal layers which give strength and rigidity to the growing leaf blades and deter feeding by chewing insects. 


\section{Pre-existing biochemical defence}

Plants liberate different chemicals, which interfere with activities of the pathogen and pathogenesis, thereby preventing or reduce infection. These chemicals and the biochemical conditions that develop may act either directly through toxic or lytic effect on the invader or indirectly through stimulating antagonistic plant surface microflora. The compounds pre-existing in plants as constitutive antibiotics and those, which are formed in response to wounds as wounds antibiotics.

Anti-microbial compounds: Plants while growing and developing release gases as well as organic substances, from leaves and roots (leaf and root exudates), containing sugars, amino acid, organic acids, enzymes, glycoside etc. These materials have profound effect on the nature of surrounding environment, particularly the phyllosphere, rhiizosphere microflora and fauna. Although these substances are ideal nutrients for microbes and help in germination and growth of several saprophytes and parasites, number of inhibitory substances is also present in these exudates. These inhibitory substances directly affect the microorganism, or encourage certain groups to dominate the environment and function as antagonists of the pathogen $[12,13]$.

Terpenoids such as of the monoterpenoids and sesquiterpenoids which are primary components of essential oils, are highly volatile compounds that contribute to the fragrance (essence) of plants that produce them. Essential oils often function as insect toxins and many protect against fungal or bacterial attack. Mint plants (Mentha spp.) produce large quantities of the monoterpenoids menthol and menthone which are produced and stored in glandular trichomes on the epidermis. Examples of such terpenoids and their sources include peppermint and spearmint (Mentha spp.), basil (Ocimum spp.), oregano (Origanum spp.), rosemary (Rosmarinus spp.), sage (Salvia spp.), savory (Satureja spp.), thyme (Thymus spp.), black pepper (Piper spp), cinnamon (Cinnamomum spp), and bay leaf (Laurus spp) [12].

Pyrethrins are monoterpenoid esters produced by chrysanthemum plants that act as insect neurotoxins. Many commercially available insecticides are actually synthetic analogues of pyrethrins, called pyrethroids, including the insecticides permethrin and cypermethrin. Pine tree resin contains large quantities of the monoterpenoids alphaand beta-pinene, which are potent insect repellents; these compounds give the organic solvent turpentine its characteristic sharp odor $[12,13]$.

Diterpenoids such as gossypol produced by cotton (Gossypium hirsutum) has strong antifungal and antibacterial properties. Phytoectysones, a triterpoenoid are mimics of insect molting hormones. When produced by plants such as spinach (Spinacia oleracea), they disrupt larval development and increase insect mortality. Limonoid triterpenoids responsible for the the fresh scent of lemon and orange peels, azadirachtin from the neem tree (Azadirachta indica) and citronella from lemon grass (Cymbopogon citratus) have antibacterial and insect repellant activities even in low concentrations as few parts per million [2].

Saponins are glycosylated triterpenoids (triterpenoids with attached sugar groups) that are present in the cell membranes of many plant species. They have detergent (soap-like) properties and act by disrupting the cell membranes of invading fungal pathogens. For instance, the wheat pathogen Gaeumannomyces graminis is unable to infect oats that contain avenacins, a class of triterpenoid saponins.

Alkaloids are a large class of bitter-tasting nitrogenous compounds that are found in many vascular plants and include caffeine, cocaine, morphine, and nicotine. They are derived from the amino acids aspartate, lysine, tyrosine, and tryptophan, and many of these substances have powerful effects on animal physiology [3]. Caffeine and theobromine are alkaloids found in plants such as coffee (Coffea arabica), tea (Camellia sinensis), and cocoa (Theobroma cacao). They are toxic to both insects and fungi. In fact, high levels of caffeine produced by coffee seedlings can even inhibit the germination of other seeds in the vicinity of the growing plants, a phenomenon called allelopathy. Allelopathy allows one plant species to "defend" itself against other plants that may compete for growing space and nutrient resources. Members of the nightshade family (Solanaceae) produce many important alkaloid compounds. Nicotine is an alkaloid that is produced in the roots of tobacco plants (Nicotiana tabacum) and transported to leaves where it is stored in vacuoles $[3,12,13]$. It is released when herbivores graze on the leaves and break open the vacuoles [3].

Cyanogenic glycosides are a particularly toxic class of nitrogenous compounds that break down to produce hydrogen cyanide (HCN), a lethal chemical that halts cellular respiration in aerobic organisms. Plants that produce cyanogenic glycosides also produce enzymes that convert these compounds into hydrogen cyanide, including glycosidases and hydroxynitrile lyases, but they are stored in separate compartments or tissues within the plant; when herbivores feed on these tissues, the enzymes and substrates mix and produce lethal hydrogen cyanide. Glucosinolates, also known as mustard oil glycosides, are sulfur-containing compounds synthesized by members of the mustard family (Brassicaceae) and produce cyanide gas when broken down by enzymes called thioglucosidases [4].

Toxic inhibitors: In many host-parasite interactions, pre-existing toxic substances in the cells form the basis of resistance. In resistant variety these substances life in abundance while in susceptible variety they may be less or completely absent. Several phenollic compounds, tannins and some fatty acid like compounds such as dienes pre-exisitg in high concentrations in cells have been implicated for the resistance or young tissues to parasitic fungal genera such as Botrytis. Many such compounds are potent inhibitors of many hydrolytic enzymes. Several other types of preformed compounds such as saponins (glycosylaled steroidal or triterpenoid compound) tomatine in tomato and avenacin in oats, have antifungal membranolytic activity (Hammond-Kosack and Jones, 1997). The fungal pathogens which lack enzymes (saponinases) that breakdown the saponins are prevented from infecting the host. Several preformed plant proteins have been reported to act as inhibitors of pathogen proteinases or of hydrolytic enzymes. Similarly lactins (proteins that bind to certain sugars) cause lyses and growth inhibition of many fungi. Plants surface cells also contain variable amounts of hydrolytic enzymes such as glucanases and chitinases, which may cause breakdown of pathogen cell wall components $[3,5]$.

Atropine is a neurotoxin and cardiac stimulant produced by the deadly nightshade plant (Atropa belladonna). Although it is toxic in large quantities, it has been used medicinally by humans in small amounts as a pupil dilator and antidote for some nerve gas poisonings. Capsaicin and related capsaicinoids produced by members of the genus Capsicumare the active components of chili peppers and produce their characteristic burning sensation in hot, spicy foods [19].

Phytoanticipins: Phytoanticipins are compounds produced during normal plant growth which may be excreted into the rhizosphere or phylloplane, accumulate in dead cells or they may be sequestered in vacuoles in an inactive form that inhibit the development of pathogens. Examples include saponins and, avenacin of oats (Avena sativa L.) provide resistance to host cells. Dead cells of brown onion skins contain the quinones catechol and protocatechuic acid, which inhibit 
germination of spores of the smudge pathogen within its rhizosphere Steroids, glycoalkaloids and glucosinalates, the latter being stored during normal growth and converted by myrosinases to active defence compounds are other examples of phytoanticipins [5].

Phytohormones: Production of the phytohormones auxins (e.g. indole-3-acetic acid-IAA) and cytokinins are important virulence factors for the gall-forming phytopathogenic bacteria, Pantoea agglomerans pv. gypsophilae, which causes crown and root gall disease of Gypsophila paniculata, and the pvs. savastanoi and nerii of Pseudomonas savastanoi, which causes olive and oleander knot disease $[2,5]$.

Phytohormones also play a very important role systemic signaling that facilitates resistance against phytopathogens. For instance, a balance between salicylic acid, a local and systemic signal for resistance against many biotrophs, and the combination of jasmonic acid and ethylene accumulation sustain signals that promote defence against necrotrophs. Additional plant hormones are likely to alter the salicylicacid-jasmonic-acid/ethylene signalling balance $[2,33,40]$.

Mamps-triggered immunity (MTI) involves repression of auxin responses, mediated in part by a micro-RNA that is also induced during abscisic-acid-mediated stress responses $[2,24,25]$. Gibberellin is produced by the fungal pathogen Gibberella fujikuroi leading to 'foolish seedling' syndrome, and cytokinin produced by many pathogens can promote pathogen success through retardation of senescence in infected leaf tissue [2]. There is therefore an interplay between MTI and normal hormone signalling, in plant immunity against pathogens [2].

\section{Plant Response to Pathogens and Response Mechanisms}

Plant responses to the presence of pathogens can be induced or active both of which involves biochemical, or cellular level defences and defences at tissue level and be specific and non-specific. The receptor-proteins are strategically located in cell membrane to detect the pathogen or factor translocated by pathogens. The ability of plant to mount an active defence response is under genomic control $[4,15]$.

\section{Induced histological defence}

After the establishment of infection in plant cells, the host defence system tries to create barriers for further colonization of tissues. This may be at various levels.

Lignifications: Lignified cell wall provide effective barrier to hyphal penetration. Lignin is a highly branched heterogeneous polymer found principally in the secondary cell walls of plants, although primary walls can also become lignified. It consists of hundreds or thousands of phenolic monomers and is a primary component of wood. Because it is insoluble, rigid, and virtually indigestible, lignin provides an excellent physical barrier against pathogen attack. Lignin acts as impermeable barrier for free movement of nutrient causing starvation of pathogen. Some examples of lignin containing crops and fungi inhibited include Peronospora parasitica, Alternaria japonica Potato: Phytophtora infestans Wheat: Septoria nodorum Cucumber: Cladosporium cucumerium, Colletorichum lagenarium Carrot: Botrytis cineria [2].

Suberization: In several plants the infected cells are surrounded by suberized cells. Thus, isolating them from healthy tissue. Corky layer formation is a part of natural healing system of plants. e.g. common scab of potato and rot of sweet potato are good examples [4].

Abscission layers: It is a gap between host cell layers and devices for dropping-off older leaves and mature fruits. Plant may use this for defence mechanism also. i.e., To drop-off infected or invaded plant tissue or parts, along with pathogen. Shot holes in leaves of fruit trees is a common feature

Tyloses: The tyloses are formed by protrusion of xylem parachymatous cell walls, through pits, into xylem vessels. The size and number of tyloses physically block the vessel. The tyloses are inductively formed much ahead of infection, thus blocking the spread of pathogen. It suggests biochemical elicitors and movement of tyloses inducing facto (TIF) up the stem. e.g. Sweet potato: Fusarium oxysporum f. sp. Batatas $[1,6]$.

Gum deposition: The gums and vascular gels quickly accumulate and fill the intercellular spacis or within the cell surroundings the infection thread and haustoria, which may starve or die.

\section{Induced cellular defence}

The cellular defence structures: There are different types of cellular defence structures such as changes in cell walls which play a limited role in defence. Such observable changes in cell wall structures include; carhohydrate apposition (synthesis of secondary wall and papillae formation), callose deposition (hyphal sheathing just outside plasma lemma around the haustorium which delays contact of pathogen (Phytophythora infestans) with host cells, structural proteins, induced cytoplasmic defence that present last line of host defence which may be effective against slow growing pathogens, weak parasites or some symbiotic relationship [5].

Papillae are callose deposits made up of polysaccharide polymers a reinforcement composed of a branched $\beta-1,3$ glucan, callose, along with silicon, lignin and proteins, between the host cell wall and plasma membrane, directly under the penetration peg. The rapid deposition of papillae is a common response of cereals to attempted penetration of epidermal cells by the powdery mildew fungus (Blumeria graminis). Papillae in resistant cultivars form more rapidly and are more diflicult to penetrate, than those formed by susceptible cultivars thereby preventing haustorial development thereby impeding cellular penetration at the site of infection [3].

Induced biochemical changes: The induced biochemical changes in host plants are the last line of host defence. This may condition a plant or plant tissue from susceptible to resistant to immune status as per their genetic potential. The role of biochemical factors in host defence is based on four main attributes which include, i) association of the substance protection against disease at the site where protection occurs, ii) isolation of the substance from the host showing protection against the disease, iii) conferment of protection by the isolated substance when introduction into to the appropriate susceptible host and, iv) resemblance of the nature of protection induced to the natural agents of a resistant plant $[1,6]$.

There are two major layers of biochemically induced plant immunity. These comprises; pathogen-associate molecular patterns (PAMPs)-triggered immunity (PTI) and effector-triggered immunity (ETI). PTI is initiated by the perception of microbe conserved PAMPs, such as bacterial flgellin and elongation factor Tu (EF-Tu), with specific plant cell. surface pattern-recognition receptors (PRRs),. PTI usually activates some early resistance responses, including stomatal closure, activation of mitogen-activated protein kinase (MAPK) cascades, transcription of resistance-related genes, reactive oxygen species (ROS) production and callose deposition. ETI on the other hand was thought to a form of accelerated and amplified PTI response. It is activated by plant intracellular resistance $(\mathrm{R})$ genes (proteins) after specific 
perception of pathogenic T3SEs, and is associated with programmed cell death, a response which is referred to as the hypersensitive response (HR) $[20,41]$.

Pathogen T3SEs are like double-edged swords; triggering ETI to display aviruelnce (Avr) functions in plant with their corresponding $\mathrm{R}$ genes on one hand, but exhibiting virulence activities in plants in absence of the corresponding $\mathrm{R}$ genes on the other. Note that that the virulence functions of most pathogen T3SEs are performed by suppressing the plant PTI and ETI [20]. It is activated by plant intracellular resistance (R) genes (proteins) after specific perception of pathogenic T3SEs, and is associated with programmed cell death, a response which is referred to as the hypersensitive response. Pathogen T3SEs are like doubleedged swords. They trigger ETI to display aviruelnce (Avr) functions in plant with their corresponding $\mathrm{R}$ genes on one hand, but exhibit virulence activities in plants in absence of the corresponding $\mathrm{R}$ genes on the other. Note that that the virulence functions of most pathogen T3SEs are performed by suppressing the plant PTI and ETI. For instance, Pto an R gene from tomato species confers a gene-for-gene resistance to Pseudomonas syringae containing AvrPto, a triggered resistance which is actually initiated by the nucleotide binding leucinerich repeat (NB-LRR) type R protein Prf, which can monitor Pto, the direct target of AvrPto [20,21]. Zimaro et al. [21] also reported that plants have evolved $\mathrm{R}$ genes/proteins which typically are intracellular receptor proteins of the NB-LRR type that recognize effectors in the cytoplasm thereby activating the effector-triggered immunity (ETI). Typically, the ability to trigger ETI is pathogen, strain or race-specific and is associated with programmed cell death, a response that is referred to as the hypersensitive response (HR), and systemic acquired resistance (SAR) in the host [21].

Toxic substances produced: Rapid production/suitable modifications and/or/accumulation of chemicals toxic to pathogen upto effective concentrations is an important component of overall active defence strategy of plants. Slow production or accumulation or low levels of similar chemicals have reported in susceptible host plants also [2].

Phenolic compounds: The phenolic compounds, viz., chlorogenic acid caffeic acid and ixidation products of floretin, hydroquinone hydroxyquionones and phytoalexins are main toxi chemical produced to inhibit pathogen or its activities. Some of these are performed toxic chemicals while others may be de novo synthesized or modified to more toxic forms. The enzymes involved in chemical pathways are present in host cell (pre-existing) [2].

Phytoalexins: Generally, plants commonly respond to stress, of either biotic (phytoalexins/insects) or abiotic (wounding) origin, by production and accumulation of substrates that can inhibit the growth and activities of the biotic factors or those that may help in the healing process [42]. One of such response is the production of phytoalexin antibiotics in the plant-pathogens interaction process or as a result reposnse to injury or other psychological simulation. They are synthesized by either the cells adjacent to the infection site, the infected host cells or by the invading pathogen. It is thought that such infected cells produce some sort of signals which induces the adjacent cells to produce the phytoalexins, which are packaged in lipid vesicles and exported to the infected cell [43]. Consequently, the infected cell becomes a toxic micro-environment for the invading pathogen. Phytoalexin accumulation is often associated with hypersensitive cell death. Examples include medicarpin (alfalfa, Medicago sativa), rishitin (tomatoes and potatoes (the Solanaceae family), and camalexin, (Arabidopsis thaliana) [2,33].
Proteins and enzymes synthesized: Plant cell walls contain proteins and enzymes that actively work to reshape the wall during cell growth and also thicken and strengthen the wall during induced defense. Post-infectional changes in host cells involve production and modification of large number of proteins (structural and enzymatic), which have important role in defence mechanism. The enzymes are required for various synthetic pathways (normal or modified) for production of resistance related substances. In addition, phenoloxidizing enzymes have vital role. The influence of these changes may be confined to infection site or nearby cells. Some plants and seeds also contain proteins and enzymes that specifically inhibit pathogen and pest enzymes.

However, unlike phytochemicals such as terpenoids, phenolics, and alkaloids, proteins require a great deal of plant resources and energy to produce; consequently, many defensive proteins are only made in significant quantities after a pathogen or pest has attacked the plant. Once activated, however, defensive proteins and enzymes effectively inhibit fungi, bacteria, nematodes, and insect herbivores. Some of the proteins and enzymes include, defensins, amylase inhibitors, lectins. ricin, protease inhibitors, hydrolytic enzymes, chitinases, and glucanases, lysozymes and hydroxyproline-rich glycoproteins $[1,6]$.

Defensins are small cysteine-rich proteins that display broad antimicrobial activity and were first isolated from the endosperm of barley (Hordeum vulgare) and wheat (Triticum aestivum). They are widely distributed and may be present in most plants. Defensins are best characterized in seeds, but can be found in virtually all types of plant tissues including leaves, pods, tubers, fruit, roots, bark, and floral tissues. They exhibit a wide range of biological activities that serve to inhibit the growth of many fungi and bacteria. Some defensins also inhibit digestive proteins in herbivores. They act by inhibiting pre-existing ion channels or form new membrane pores that disrupt cellular ion balance and as proteinase and polygalacturonase-inhibitors, as ribosome inhibitors or lectins thereby interfering with pathogen nutrition retarding their development, thus contributing to plant disease resistance $[12,13]$.

Lectins are non-enzymatic proteins and glycoproteins that bind to carbohydrates and exhibit a wide range of functions including disruption of digestion in insects and agglutination of blood cells in vertebrates.

Ricin is a very highly potent toxin produced in castor beans (Ricinus communis), having an average lethal dose of only 0.2 milligrams in humans. It combines a lectin molecule with an $\mathrm{N}$-glycoside hydrolase that enters animal cells and inhibits protein synthesis [3].

Protease inhibitors are typically produced in response to herbivore attack and inhibit digestive enzymes including trypsin and chymotrypsin. They occur widely in nature but have been well studied in legumes, solanaceous plants, and grasses. Herbivore feeding often triggers a series of molecular signaling events that induce systemic production of these compounds in distal tissues that contribute to the protection of undamaged plant parts from subsequent attacks by a wide range of herbivore pests $[3,43]$.

Hydrolytic enzymes are produced by some plants in response to pathogens and often accumulate in extracellular spaces where they degrade the cell walls of pathogenic fungi $[3,15]$.

Chitinases are enzymes that catalyze the degradation of chitin, a polymer with a backbone similar to cellulose that is present in the cell walls of true fungi [3].

Glucanases are enzymes that catalyze the degradation of glycosidic 
linkages in glucans, a class of polymers similar to cellulose that is present in the cell walls of many oomycetes (water molds). In vitro analysis has verified the anti-fungal properties of these compounds, and transgenic plants expressing high levels of these enzymes exhibit increased resistance to a wide range of both foliar and root pathogens [3]. Lysozymes are hydrolytic enzymes that are capable of degrading bacterial cell walls [3].

Hydroxyproline-rich glycoproteins are structural proteins in plant cell walls involved in the organization of secondary cell wall thickening. Genes encoding hydroxyryproline-rich glycoprotein biosynthesis are transcribed in advance of invading hyphae, making cell walls tougher. Hydrogen peroxide, released during the oxidative burst following pathogen challenge, causes extensive cross-linking between hydroxyproline-rich glycoproteins and other cell wall components, making the walls even more resistant to microbial digestion [3]. Generally the proteins and enzymes act by forming complexes that block active sites or alter enzyme conformations, ultimately reducing enzyme function [2].

Inactivation of enzymes and toxins: The role played by chemical weapons (toxin and enzymes) of pathogens during pathogenesis is well established. The necrotrophs and hemihiotrophs employ more of these substances fro causing those tissue damage as compared to speacialized obligate parasites. The defence strategy of resistant plants, through activity of phenols, tannins and protein as enzymes inhibitors, the phenolics are not anti-fungal but make pathogen ineffective by neutralizing their enzymes. In immature grape fruits catechol-tannin is known to inhibit enzymes produced by Botrytis cinerea. Toxins are known to be involved in pathogenesis to various edtents (pathotoxins/ vivotoxins). The resistance to toxins, in host, will be resistance to pathogens. This can be achieved by detoxification or lack of receptor sites for these toxins [2].

Alteration of biosynethetic pathway: The pose inflectional metabolism of host tissue is altered (stress physiology) to cope with the advancing activities of pathogen. New enzymes (proteins) are produced in an effort to synthesize defence related substances. Most of these compounds are formed through Shikmic acid pathway and modified acetate pathway. Respiration in diseased tissue is invariably increased; a part of glycolysis is replaced by pentose pathway, which yields four carbon compounds are formed through Shikmic acid pathway and modified acetate pathway. Respiration in diseased tissue is invariably increased; a part of glycolysis is replaced by pentose pathway, which yields four carbon compounds. It is possible that in early stages of infection the gene regulation of host cell is influenced and some specific genes [4].

\section{Pathogen Recognition in Plants}

For plants to effectively control invading pathogens, they must recognize such pathogens as foreign non-host components. Consequently they have evolved very efficient signaling mechanisms that enable them to detect microbial pathogens. Pathogen recognition is often triggered following the perception of intruding microbes or their products by more or less generic microbe or pathogen associated molecular patterns (MAMPs or PAMPs). These are certain class of plasma membrane bound extracellular receptors also called Pattern Recognition Receptors (PRRs) capable of detecting or recognizing microbial elicitors. In the presence of MAMPs, PRPs are activated leading to production of active defense responses against these pathogens. This ensures that the would-be pathogens are stopped before they could cause any serious infection in the plant [44]. Examples of such PRPs include chitin, oligogalacturonides, ergosterol, bacterial flagellin, Pep-13, xylanase, cold-shock protein and lipopolysaccharides (LPS). Pathogen recognition is a non-specific process and therefore more accurately referred to as PAMP triggered immunity (PTI). Such MAMPs or PAMPs none-self recognition by plants is triggered by the help of elicitors; non-specific and specific elicitors [43].

\section{Non-specific elicitors}

These are abiotic elicitors, including heavy metal ions, UV light and some metabolic inhibitors, precipitate physiological stress responses, some of which contribute to resistance. Their effect is generally transitory and non-specific $[2,3,12,13]$.

\section{Specific elicitors}

These are mostly biotic elicitors whose actions might be targeted or specific and they include secondary metabolites with far more fatal effects. Different categories of specific elicitors include:

Damage-Associated Molecular Patterns (DAMPs): Many plant pathogens such as Phgtophthora megasperrna f. sp. glocinea of soybeans produce lytic enzymes (e.g. polygalacturonase released by fruit decay fungi and bacteria), cell wall fragments (heptabetaglucan), cutin monomers and peptides which obstruct, destroy or dissolve the structural barriers such as the middle lamela of plant tissues. These products function as endogenous elicitors called Damage-Associated Molecular Patterns (DAMPs) and characteristically emerge in the apoplast and serve as danger signals to induce plant innate immunity $[2,33]$.

'Elicitins' are a group of $10 \mathrm{kD}$ peptides from Phytophthora spp. and a number of related oomycetes. Polyunsaturated fatty acids like arachidonic and eicosapentaenoic acid from cell membranes of Phgtophthora infestans elicit defence responses in potatoes.

Gene-specific elicitors are elicitors that are induced by avirulence genes in the pathogen. Some specific disease resistance $(R)$ genes help in recognizing pathogens.

Insect related elicitors found specifically in saliva of chewing insects that enables plants to distinguish between general wounding and insect feeding [9].

Volatile organic compounds (VOCs) are organic secondary metabolites including monoterpenoids, sesquiterpenoids, and homoterpenoids, released by plants in response to pathogen invasion. These chemicals may repel harmful insects or attract beneficial predators that prey on the destructive pests [9]. For example, wheat seedlings infested with aphids may produce VOCs that repel other aphids. Lima beans and apple trees emit chemicals that attract predatory mites when damaged by spider mites, and cotton plants produce volatiles that attract predatory wasps when damaged by moth larvae. Feeding on one part of the plant can induce systemic production of these chemicals in undamaged plant tissues, and once released, these chemicals can act as signals to neighboring plants to begin producing similar compounds. Production of these chemicals exacts a high metabolic cost on the host plant, so many of these compounds are not produced in large quantities until after insects have begun to feed [3].

\section{The chemistry of Plant Immune Responses}

\section{Alkalinization of medium}

Plants do not have defined cell mediated and antibody mediated immune responses as in animals. Most of the defence responses in 
animals has to do ionic changes, production of secondary metabolites are development of physical barriers against infections [9]. The initial defense response elicited by plant cell cultures in response to MAMPs is the alkalinization of the growth medium due to immediate changes in the concentrations of $\mathrm{H}^{+}, \mathrm{K}^{+}, \mathrm{Cl}^{-}$, and $\mathrm{Ca}^{2+}$ ions across the plasma membrane which occurs immediately after elicitation. Elevation of $\mathrm{Ca}^{2+}$ leads to their accumulation in the thylakoid lumen of chloroplasts followed by an increased influx in the cytoplasm [9]. $\mathrm{Ca}^{2+}$ elevation in cytosol plays a pivotal role in mediating other plant immune processes, including control of reactive oxygen species (ROS), salicylic acid (SA) production and stomatal closure [6]. A high extracellular calcium $\left(\mathrm{Ca}^{2+o}\right)$ level induces $\mathrm{H}_{2} \mathrm{O}_{2}$ and $\mathrm{NO}$ accumulation in guard cells, which further triggers $\mathrm{Ca}^{2+\mathrm{i}}$ transients and finally results in stomatal closure. The decrease in photosynthesis in response to infection is another defense strategy employed by plants to dodge the pathogens [6].

\section{Nitrosative burst and oxidative burst}

Nitrosative burst is the production of reactive nitrogen intermediates (RNIs) and oxidative burst is the production of reactive oxygen species (ROS) at the cell surface. These mechanisms are one of the earliest events detectable attributing to plant defense. The ROS synthesis pathway has been well studied and understood but RNI remain largely unclear. Earlier reports indicate that there is covalent attachment of the nitric oxide (NO) moiety to a protein cysteine thiol to form an S-nitrosothiol (SNO), a redox-based post-translational modification, which proceeds through a cascade of biochemical pathways to produce RNI [1,6]. During higher concentrations of S-nitrosothiols, the NO directs a negative feedback loop mediated by Snitrosylation of the NADPH oxidase (at Cys 890), thus restricts the HR by inhibiting the synthesize of ROS. The cys 890 was reported to be evolutionarily conserved and exclusively S-nitrosylated in both human and Drosophila NADPH oxidase, signifying that this system may govern immune responses in both plants and animals. ROS are potentially toxic analogous of reduced oxygen forms, such as the superoxide anion and hydrogen peroxide. They are considered to exert antimicrobial action through in direct microbicidal actions, strengthening of the cell wall through oxidative cross-linking of glycoproteins, induction of intracellular signalling pathway such as the synthesis of salicylic acid (SA) and activation of systemic acquired resistance (SAR) associated with systemic propagation of the oxidative burst $[15,34]$.

\section{Effector triggered immunity (ETI)}

ETI is the plant immune response events specifically targeting pathogenic mechanisms that involve transcriptional processes in the plant host nucleus. For instance, successful pathogens promote pathogenesis by injecting a battery of effector proteins across the plant cell wall into the cytoplasm and subsequently the nucleus. These injected effector proteins or Avr proteins which are avirulent in nature contributes to pathogen virulence in three ways; i) they act as transcription factors that directly activate transcription in host cells, (ii) they affect histone packing and chromatin configuration, and/ or (iii) (they) directly target host transcription factor activity and ultimately promote the release of nutrients required for the survival of pathogen $[10,15]$.

Plant ETI therefore respond to this sort of infection by activation of downstream transcription factors which include a string of pathogenesis related (PR) genes in and around the infected cell for the biosynthesis of chemical substances in order to limit the infection. Chemical substances biosynthesized for this purpose include salicylic acid (SA), jasmonic acid (JA), ethylene (ET), production of various antimicrobial compounds in endoplasmic reticulum and their secretion into vacuoles as well as cell-wall strengthening and lignification. Salicylic acid thus accumulated in the infected areas binds to the receptor NPR3 of the $\mathrm{PR}$ genes in order to mediate the degradation of the infected cell by a process similar to the programmed cell death (apoptosis) found in animals $[1,6]$. Salicyclic acid accumulation is accompanied by the development of hypersensitive response (HR).

Hypersensitive response (HR) is responsible for restricting the bacteria and for blocking bacterial multiplication in planta. HR and necrotic responses impart resistance against diverse plant pathogenic fungi, bacteria, and viruses, and, to some extent, use similar mechanisms [41]. It is however difficult to establish which defense responses occur during HR, because it involves a complex combination of responses, which may act additively or synergistically [3]. Once HR has been triggered, plant tissues may become highly resistant to a broad range of pathogens for an extended period of time. This phenomenon is called systemic acquired resistance ( $S A R$ ) and represents a heightened state of readiness in which plant resources is mobilized in case of further attack [3]. HR in turn leads to a form of programmed cell death (PCD) characterized by cytoplasmic shrinkage, chromatin condensation, mitochondrial swelling, vacuolization and chloroplast disruption [6].

There are two main classes of PCD namely autolytic-PCD and nonautolytic PCD. Autolytic-PCD involves a rapid cytoplasm clearance after tonoplast rupture due to the release of hydrolases from the vacuole, which degrades the cytoplasm and brings about a localized cell death. This autolytic-PCD is associated with increased caspase-like activities. Caspases are a family of cysteine proteases that cleave their substrates after an aspartic acid residue activates other caspases and degradative enzymes or proteins involved in necrosis. Non-autolytic PCD involves the absence of a rapid clearance of the cytoplasm even in the persistence of increased permeability of tonoplast. This increased permeability or even rupture of tonoplast does not result in considerable discharge of hydrolases, which instantaneously clear the remaining cytoplasm. In The non-autolytic PCD mechanism the plasma membrane fuses with the membranes of a large central vacuole mediated by proteasome subunit, resulting in the release of vacuolar antibacterial proteins in the apoplast. The extracellular fluid discharged has both antibacterial and cell death-inducing activity thereby promoting local necrosis and ultimately obstructing the pathogen growth $[6,45]$.

\section{Bacterial Virulence Factors}

The severity of disease symptoms caused by bacteria in plants is determined by the production of a number of virulence factors, including phytotoxins, plant cell-wall-degrading enzymes, extracellular polysaccharides and phytohormones. In general, phytopathogenic bacterial strains mutated in any virulence factor more or less reduce their virulence, while their pathogenicity remains unchanged [3].

\section{Phytotoxins}

Phytopathogenic bacteria produce a wide spectrum of nonhostspecific phytotoxins, i.e., toxic compounds causing symptoms in many plants. For instance, based on the symptoms they induce in plants, phytotoxins of Pseudomonas spp. have been grouped ito necrosisinducing and chlorosis-inducing phytotoxins.

Necrosis-inducing phytotoxins such as lipodepsipeptides are grouped into two based on their amino acid chain length: mycins (e.g. syringomycins) and peptins (e.g. syringopeptins). Both phytotoxins induce necrosis in plant tissues and form pores in plant plasma 
membranes, thereby promoting transmembrane ion flux and cell death [45].

Chlorosis-inducing phytotoxins include coronatine, produced by P. syringae pvs. atropurpurea, glycinea, maculicola, morsprunorum and tomato, phaseolotoxin, produced by Pseudomonas savastanoi pv. phaseolicola and $P$. syringae pv. actinidiae, and tabtoxin produced by the pvs. tabaci, coronafaciens, and garcae of $P$. syringae. Coronatine synthesis contributes significantly to lesion expansion, the development of chlorosis, and bacterial multiplication in infected leaves. It is also able to suppress stomatal closure in Arabidopsis a process which is part of the plant's innate immune response, which prevents bacteria from entery, The compound prevents this closure re-opening it thereby granting microbial access into the tissues of the plant [3].

\section{Plant cell-wall degrading enzymes}

Extracellular enzymes able to degrade plant cell walls are essential virulence factors for necrotrophic soft rot bacteria, such as the soft rot erwiniae, now belonging to the Pectobacterium genus (e.g. Pectobacterium carotovorum subsp. carotovorum, Pectobacterium atrosepticum, Pectobacterium chrysanthemi). A combination of extracellular enzymes: pectate lyases, pectin methylesterases, pectin lyases, polygalacturonases, cellulase, and proteases are involved in the depolymerization process of plant cell walls provoked by these bacteria $[1,6]$.

\section{Extracellular polysaccharides}

Extracellular polysaccharides (EPSs) may be associated with the bacterial cell as a capsule, be released as fluidal slime, or be present in both forms [45]. EPSs are important pathogenicity or virulence factors, particularly for bacteria with a vascular habitat. For example, the EPSs amylovoran and levan are pathogenicity and virulence factors, respectively, of Erwinia amylovora, the causal agent of fire blight on some Rosaceous plants. Amylovoran affects plants primarily by plugging the vascular tissue, thus inducing wilt of shoots, and is considered a pathogenicity factor. Xanthan, the major exopolysaccharide secreted by Xanthomonas spp., suppresses callose deposition in plant cell wall, a basal form of resistance to bacterial colonization $[3,12,13]$.

\section{Defense against Viral Infections}

During a viral infection, in a manner similar to nonviral infections, an HR response is initiated by Avr/R protein interactions that lead to metabolic changes in defense hormone levels, such as salicylic acid (SA), jasmonic acid (JA), and nitric oxide (NO), and the accumulation of reactive oxygen species (ROS), such as $\mathrm{O}_{22}$ and hydrogen peroxide, both in the infected and noninfected tissues. At the cellular level, HR affects calcium $\left(\mathrm{Ca}^{2+}\right)$ ion homeostasis and alters membrane potential and permeability while, at the molecular and biochemical level, several genetic signaling cascades are activated during HR to induce multiple proteins, including mitogen-activated protein (MAP) kinase proteins Downstream of these primary signaling cascades, expression of several defense related proteins, such as glucanases, chitinases, and defensins, in the pathogenesis-related protein family are upregulated. Remarkably, the genetic components that mediate HR against diverse viral and nonviral pathogens are conserved across plant genera. At the molecular and biochemical level, several genetic signaling cascades are activated during HR to induce multiple proteins, including mitogenactivated protein (MAP) kinase proteins. Downstream of these primary signaling cascades, expression of several defenserelated proteins, such as glucanases, chitinases, and defensins, in the pathogenesis-related protein family are upregulated. Remarkably, the genetic components that mediate HR against diverse viral and nonviral pathogens are conserved across plant genera $[20,46]$.

Recently, a jacalin-type lectin, JAX1, was shown to confer resistance against multiple potexviruses (PVX, PLAMV, White clover mosaic virus, and Asparagus virus 3). Although JAX1 and RTM1 are closely related lectin proteins, they impart distinct forms of resistance. JAX1 functions at the cellular level, inhibiting replication of PLAMV, and RTM1 inhibits systemic movement of TEV. An intriguing aspect of lectin-mediated resistance (LMR) compared with the NB-LRR resistance is that it does not invoke HR and SAR responses, nor does it alter SA levels, signaling, or other typical defense gene expression changes commonly modulated in immune resistance responses [46].

The ubiquitin proteasome system (UPS) has emerged as a prominent player in influencing virus-host interactions at almost every stage of antiviral defense, both in plants and viruses use a plethora of strategies to modulate UPS processes. UPS regulates cellular activities including cell cycle, transcription, and signal transduction. The plant UPS primarily involves the activity of ubiquitin-activating enzyme (E1), ubiquitinconjugating enzyme (E2), and ubiquitin-ligase (E3). These three proteins form the E3 ubiquitin ligase Viruses and Host Immune Responses 1495 complex that specifically polyubiquitinates cellular proteins that are subsequently targeted for degradation by the $26 \mathrm{~S}$ proteasome [46].

In addition to the hypersensitive response, plants can defend themselves against viruses by a variety of mechanisms including a sophisticated genetic defense system called RNA silencing $[3,12,13]$. Many viruses produce double-stranded RNA or DNA during replication in a host cell. Plants can recognize these foreign molecules and respond by digesting the genetic strands into useless fragments and halting the infection. Plants that are infected with viruses will often exhibit chlorosis and mottling, but disease symptoms may eventually disappear if RNA silencing is successful, a process called recovery [3]. In addition, the plant may retain a template of the digested genetic strand that can be used to quickly respond to future attack by similar viruses, a process analogous to the memory of vertebrate immune systems $[1,3]$.

\section{Conclusion}

In order to effectively enhance a virile and robust plant agricultural turn over, and to have effective pathogen control policy and strategy, it is important that what causes pathogen growth arrest be well studied. Because plants are sessile, they must continuously integrate both biotic and abiotic signals from the environment. Unfortunately plants lack circulating cells resulting in partitioning of the signal responses both locally over several cell diameters and systemically in a limited sphere. This therefore highlights the need to adequately understand the cell signaling mechanisms and the role of hormones in disease resistance for adequate control of plant diseases.

\section{References}

1. Jan AT, Azam M, Ali A, Haq Q (2011) Novel approaches of beneficial Pseudomonas in mitigation of plant diseases - an appraisal. Journal of Plant Interactions 6: 195-205.

2. Jones JD, Dangl JL (2006) The plant immune system. Nature 444: 323-329.

3. Freeman BC, Beattie GA (2008) An Overview of Plant Defenses against Pathogens and Herbivores. The Plant Health Instructor.

4. Van Baarlen $P$, Van Belkum A, Summerbell RC, Crous PW, Bart P, et al. (2007) Molecular mechanisms of pathogenicity: how do pathogenic microorganisms develop cross-kingdom host jumps? FEMS Microbiology Reviews 3: 239-227. 
5. Anderson JP, Gleason CA, Foley RC, Thrall PH, Burdon JB, et al. (2010) Plants versus pathogens: an evolutionary arms race. Funct Plant Biol 37: 499-512.

6. Dou D, Zhou JM (2012) Phytopathogen effectors subverting host immunity: different foes, similar battleground. Cell Host Microbe 12: 484-495.

7. Wen L (2012) Cell Death in Plant Immune Response to Necrotrophs. J Plant Biochem Physiol 1: e103.

8. Guttman DS, McHardy AC, Schulze-Lefert P (2014) Microbial genome-enabled insights into plant-microorganism interactions. Nat Rev Genet 15: 797-813.

9. Pusztahelyi T, Holb IJ, Pócsi I (2015) Secondary metabolites in fungus-plant interactions. Front Plant Sci 6: 573.

10. Surico G (2013) The concepts of plant pathogenicity, virulence/avirulence and effector proteins by a teacher of plant pathology. Phytopathologia Mediterranea 52: 399-417.

11. Narayanasamy $P$ (2011) Microbial plant pathogens-detection and disease Diagnosis. Fungal Pathogens 1: 5-199.

12. Brown J, Guest D (1980) Plant defences against pathogens. Australian ViceChancellors' Committee, Canberra. pp. 263-285.

13. Buonaurio $R$ (2008) Infection and plant defense responses during plantbacterial interaction. In: Barka EA. Clément C (eds) (2008). Plant-Microbe Interactions pp. 169-197.

14. Boyle EC, Finlay BB (2003) Bacterial pathogenesis: exploiting cellular adherence. Curr Opin Cell Biol 15: 633-639.

15. Piña-Vázquez C, Reyes-López M, Ortíz-Estrada G, de la Garza M, SerranoLuna J (2012) Host-parasite interaction: parasite-derived and -induced proteases that degrade human extracellular matrix. Journal of Parasitology Research pp. 1-24.

16. Fatima U, Senthil-Kumar M (2015) Plant and pathogen nutrient acquisition strategies. Frontiers in Plant Science 6: 750

17. Grennan AK (2006) Plant response to bacterial pathogens. Overlap between innate and gene-for-gene defense response. Plant Physiol 142: 809-811.

18. Zeilinger S, Gupta VK, Dahms TE3, Silva RN4, Singh HB, et al. (2015) Friends or foes? Emerging insights from fungal interactions with plants. FEMS Microbio Rev.

19. Hammond-Kosack KE, Jones JD (1997) PLANT DISEASE RESISTANCE GENES. Annu Rev Plant Physiol Plant Mol Biol 48: 575-607.

20. Hou S, Yang Y, Wu D, Zhang C (2011) Plant immunity: evolutionary insights from PBS1, Pto, and RIN4. Plant Signal Behav 6: 794-799.

21. Zimaro T, Gottig N, Garavaglia BS, Gehring S, Ottado J (2011) Unraveling Plant Re sp onses to Bacterial Pathogens through Proteomics. Journal of Biomedicine and Biotechnology 1: 1-12.

22. Vidaver AK, Lambrecht PA (2004) Bacteria as plant pathogens. The Plant Health Instructor.

23. Melotto M, Underwood W, He SY (2008) Role of stomata in plant innate immunity and foliar bacterial diseases. Annual Reviews in Phytopathology 46: 101-122.

24. Brooks DM, Bender CL, Kunkel BN (2005) The Pseudomonas syringae phytotoxin coronatine promotes virulence by overcoming salicylic aciddependent defences in Arabidopsis thaliana. Molecular Plant Pathology 6 629-640.

25. Melotto M, Underwood W, Koczan J, Nomura K, He SY (2006) Plant stomata function in innate immunity against bacterial invasion. Cell 126: 969-980.

26. Mitchell PL (2004) Heteroptera as vectors of plant pathogens. Neotropical Entomology 33: 519:545.
27. Laluk K, Mengiste T (2010) Necrotroph attacks on plants: wanton destruction or covert extortion? Arabidopsis Book 8: e0136.

28. Tabassum B, Nasir IA, Aslam U, Husnain T (2012) How RNA interference combat viruses in plants. In: Meroni, G. and Petrora, F. (eds) (2012) Functional Genomics.

29. García JA, Pallás V (2015) Viral factors involved in plant pathogenesis. Curr Opin Virol 11: 21-30

30. Martines RB, Ng DL, Greer PW, Rollin PE, Zaki SR (2015) Tissue and cellular tropism, pathology and pathogenesis of Ebola and Marburg viruses. J Pathol 235: 153-174.

31. Serrano M, Coluccia F, Torres M, L'Haridon F, Métraux JP (2014) The cuticle and plant defense to pathogens. Front Plant Sci 5: 274

32. Baroni A, Buommino E, De Gregorio V, Ruocco E, Ruocco V, et al. (2012) Structure and function of the epidermis related to barrier properties. Clin Dermatol 30: 257-262.

33. Cai K, Gao D, Chen J, Luo S (2009) Probing the mechanisms of siliconmediated pathogen resistance. Plant Signal Behav 4: 1-3.

34. Gomathi S, Ambikapathy V, Panneerselvam A (2011) Antimicrobial Activity of Some Medical Plants Against Pythium debaryanum (Hesse). Journal of Microbiology and Biotechnology Research 1: 8-13

35. Janda M, Matouskova J, Burketova L, Valentova O (2014) Interconnection between actin cytoskeleton and plant defense signaling. Plant Signal Behav 9: e976486

36. Porter K, Day B (2015) From filaments to function: The role of the plant actin cytoskeleton in pathogen perception, signaling, and immunity. J Integr Plant Biol.

37. Falcão F, Melo-de-Pinna GF, Leal IR, Almeida-Cortez JS (2003) Morphology and anatomy of extrafloral nectaries in Solanum stramonifolium (Solanaceae) Poliana. Canadian Journal of Botany 81: 859-864.

38. Glas JJ, Schimmel BCJ, Alba JM, Escobar-Bravo R, Schuurink RC, et al (2012) Plant Glandular Trichomes as Targets for Breeding or Engineering of Resistance to Herbivores. International Journal of Molecular Science 13 . 17077-17103

39. Mazen AMA, Zhang D, Franceschi VR (2004) Calcium oxalate formation in Lemna minor: physiological and ultrastructural aspects of high capacity calcium sequestration. 161: 435-448.

40. Leipe DD, Koonin EV, Aravind L (2004) STAND, a class of P-loop NTPases including animal and plant regulators of programmed cell death: multiple complex domain architectures, unusual phyletic patterns, and evolution by horizontal gene transfer. Journal of Molecular Biology 343: 1-28.

41. Mandadi KK, Scholthof KB (2013) Plant immune responses against viruses: how does a virus cause disease? Plant Cell 25: 1489-1505

42. War AR, Paulraj MG, Ahmad T, Buhroo AA, Hussain B, et al. (2012) Mechanisms of plant defense against insect herbivores. Plant Signal Behav 7: 1306-1320.

43. Mogensen TH (2009) Pathogen recognition and inflammatory signaling in innate immune defenses. Clin Microbiol Rev 22: 240-273.

44. Zipfel C, Felix G (2005) Plants and animals: a different taste for microbes? Curr Opin Plant Biol 8: 353-360.

45. DebRoy S, Thilmony R, Kwack YB, Nomura K, He SY (2004) A family of conserved bacterial effectors inhibits salicylic acid-mediated basal immunity and promotes disease necrosis in plants. Proceedings of the National Academy of Science, USA 101: 9927-9932.

46. Yamaji Y, Maejima K, Ozeki J, Komatsu K, Shiraishi T, et al. (2012) Lectinmediated resistance impairs plant virus infection at the cellular level. Plant Cell 24: 778-793. 